\title{
Comportamiento de los sedimentos fluviales en Honduras
}

\author{
Roberto Fredy Ávalos Lingán*
}

\section{RESUMEN}

El Ministerio de Recursos Naturales y Ambiente hasta 1984 manejaba una fuerte campaña de medición de sedimento en suspensión en sus principales estaciones hidrométricas. Por otra parte la Empresa Nacional de Energía Eléctrica (ENEE) manejaba también hasta los años 90 una fuerte medición de sedimento en las cuencas de su interés. La ENEE retomo en el 2000 dichas mediciones. Toda la información recolectada a la fecha presenta mucha discontinuidad en el tiempo y en frecuencia de medición. La información rescatable ha sido pocas veces investigada, al grado que los estudios sedimentológicos de los proyectos hidroeléctricos se basan en criterio de rendimiento y pérdida de suelo, según la experiencia de los consultores.

De lo mencionado se creyó necesario investigar, con la información disponible, los rendimientos y pérdida de suelo para la zona central y oriental del país. Esto permitirá disponer de alguna base científica para evaluar los valores utilizados por los consultores. La hipótesis que en el rango de rendimiento se encuentra entre 500 y 1000 (Ton/año/km²) es aceptada como resultado de la presente investigación.

\section{Palabras clave: Sedimento, sedimento en suspensión, sedimento de fondo, muestreo integral, caudal líquido, caudal sólido, relación, rendimiento y pérdida de suelo}

\section{ABSTRACT}

The Ministry of Natural Resources and Environment until 1984 ran a strong campaign measurement of suspended sediment in the main hydrometric stations. Moreover the National Electricity Company (ENEE) ran until the ' 90 s also a strong measure of sediment in the basins of interest. ENEE in 2000 has returned to these measurements. All information collected, to date, presents a lot of discontinuity in the time and frequency measurement. Callable information has seldom been investigated to the point that sediments studies of hydroelectric projects are based on performance criteria and soil loss, based on experience of the consultants. 
From the above it was thought necessary to investigate, with the information available, yields and soil loss for the central and east. This will provide a scientific basis for evaluating the values used by the consultants. The hypothesis that the performance range is between 500 and 1000 (tons /year $/ \mathrm{km}^{2}$ ) is accepted as a result of this investigation.

Key words: Sediment, suspended sediments, bottom sediments, integral sampling, flow liquid, solid flow, relation, performance and soil loss 


\section{INTRODUCCIÓN}

Cada inicio del periodo lluvioso se observa en las diferentes cuencas hidrográficas del país que las aguas de los ríos y quebradas transportan agua con una fuerte turbidez, dependiendo esto principalmente de la lluvia (cantidad, intensidad y duración), del tipo y estado del suelo, de la cobertura vegetal y de la morfología de la cuenca.

De acuerdo a la publicación realizada por la Secretaría de Recursos Naturales y Ambiente (Balance Hídrico de Honduras, 2004), a lluvia promedio en Honduras es alta comparado con la evapotranspiración potencial. El valor promedio de la lluvia es de 1880 mm/año y la evapotranspiración potencial de 1315 mm/año, calificándole como Un país de clima húmedo y subhúmedo con una fuerte riqueza hídrica.

La lluvia es el principal agente en la producción de sedimento en la cuenca y los caudales en los cursos de agua en la erosión del lecho. Tomando en cuenta la situación actual de las cuencas desde el punto de vista de cobertura vegetal y constitución y estado del suelo y lo mencionado en el párrafo anterior, el territorio hondureño se encuentra sometido a un fuerte potencial de erosión. El objetivo de la presente investigación es determinar, a partir de la información disponible, los rendimientos de sedimento en ton/año/ $\mathrm{km}^{2}$ y la pérdida de suelo en mm/año para la zona central y oriental del país. Como resultado podemos mencionar que la parte oriental de país tiene un rendimiento de 685.586 ton/año $/ \mathrm{km}^{2} \mathrm{y}$ una pérdida de suelo de $0.857 \mathrm{~mm} / \mathrm{año}$, y la parte central de 843.344 ton/año $/ \mathrm{km}^{2}$ y una pérdida de suelo de $1.054 \mathrm{~mm} / \mathrm{año}$.

\section{ANTECEDENTES}

De acuerdo a la información obtenida se sabe que desde los años 60 el Departamento de Hidrología y Climatología de la Dirección de Recursos Hídricos (DGRH) del Ministerio de Recursos Naturales y Ambiente realizaba muestreos de sedimento en los principales ríos del país y que mediante su laboratorio de sedimentos, de ese tiempo, obtenía las concentraciones y la producción de sedimento. Aparentemente esto duró hasta mediados de los años 84; información de sedimento antes de esos años no fue posible obtener.

Por otra parte, la Empresa Nacional de Energía Eléctrica (ENEE) también desde los años 60 inició muestreos de sedimentos integral y puntual en sus estaciones, donde se proyectaba una central hidroeléctrica. Disponía de un laboratorio de sedimento 
para la medición de concentraciones. A la fecha actual el laboratorio se ha modernizado no solamente para concentraciones de sedimentos, sino también de calidad de agua y otras variables más. A través del tiempo los muestreos de sedimento en las estaciones hidrométrica de la ENEE disminuyeron, pero actualmente se ha retomado el plan de operaciones de muestreo. Cabe mencionar que ambas instituciones gubernamentales solamente han medido 0 miden sedimento en suspensión.

En el Estudio de Factibilidad del Motors-Columbus (1973). Proyecto El Cajón. Volumen 2. Hidrología. Suiza. Dicha empresa utilizó los datos de sedimento disponibles del rio Humuya a esa fecha y a su vez realizó algunos muestreos, llegando finalmente a determinar un rendimiento de 733.25 ton/año/ $/ \mathrm{km}^{2}$. La ENEE en 1987 realizó un análisis de sedimento de sus estaciones hidrométricas que disponían mayor información, para lo cual aplicó el método del Bureau of Reclamation, considerando las curvas de sedimento ajustadas a las Líneas de concentración establecidas por la ENEE y las curvas de duración de los caudales diarios. Lamentablemente no se pudo disponer de los datos ni del informe, pero si de algunas curva de sedimentos.

En mayo de 1991 la consultora alemana LAHMEYER, dentro de los estudios de los proyectos hidroeléctricos Remolino y Sico II, determinó para el río Patuca en la estación hidrométrica Cayetano el rendimiento de 133.0 ton/año/ $/ \mathrm{km}^{2}$, calificando este resultado como muy bajo; debido a la calidad de la información existente y a su discontinuidad. Finalmente esta empresa trabajó con rangos de rendimiento de 500 a 900 ton $/$ año $/ \mathrm{km}^{2}$.

\section{MARCOCONCEPTUAL}

La información de sedimento en muestreados por las instituciones anteriormente mencionadas, cuyos planes de muestreos quizás no fueron realizados bajo ningún criterio científico es la que actualmente se dispone, ya que a la fecha no es posible determinar en forma exacta los rendimientos y pérdida de suelo para las diferentes cuencas del país. Queda, pues por preguntarse en qué rango pueden encontrarse los rendimientos y pérdida de suelo en Honduras.

Son los proyectos hidroeléctricos que han hecho realidad las mediciones de sedimento que actualmente existen en el país. Estas mediciones son utilizadas para determinar el volumen muerto de los embalses: el especialista y el diseñador se ponen de acuerdo para discutir los resultados y proponer un valor razonable.

Conocer las pérdidas de suelo de las cuencas y de los cursos de agua es importante 
para el desarrollo sostenible y sustentable del país; recordemos que es en las primeras capas del suelo donde se encuentra la riqueza agrícola, y no mencionar sus capacidades físicas que se ponen en riesgo. Esto permitirá establecer científicamente planes operativos urgentes para la conservación de los suelos.

\section{HIPÓTESIS}

La hipótesis a probar es: Para la zona Central y Oriental los rendimientos se encuentran entre 500 y 1000 ton $/ \mathrm{año} / \mathrm{km}^{2}$.

\section{METODOLOGÍA}

En base a la información disponible las muestras para la zona central son representadas, en esta investigación, por la información disponible de los ríos Malapa, Guacamaya y Maragua, de sus estaciones hidrométricas correspondientes, tomando en consideración únicamente las muestras obtenidas por muestreo integral. Para el caso de la zona oriental se pudo contar con los datos de sedimento de muestreo integral del río Patuca, justo después de la unión de los ríos Guayape y Guayambre en la estación hidrométrica Cayetano. Cabe mencionar que las estaciones hidrométricas utilizadas pertenecen y son manejadas por la ENEE. El plano 1 muestra la ubicación de los sitios de muestreo.

La calidad de la realización de los muestreos y los análisis de laboratorio están regidos por normas internacionales, en especial por la Organización Mundial Meteorológica y por parte de los fabricantes de equipo. En cuanto a la frecuencia de muestreo, las normas dicen que hay que realizarlos durante todo el año, pero con una frecuencia mayor en los meses lluviosos debido a que son los tiempos de mayor sedimento, sin embargo la frecuencia del número de muestreos, no esta definida.

Cabe señalar que no se dispone de una serie con un número de datos uniformes de sedimento en suspensión en cada estación hidrométrica mencionada. Los análisis a aplicar serán realizados para el periodo disponible de cada una de ellas. Para determinar los rendimientos y pérdida de suelo de las cuencas con información disponible la metodología consistirá en encontrar alguna relación entre los caudales líquidos QL ( $\mathrm{m}^{3} / \mathrm{s}$ ) y sólidos QS (ton/día) justo en el periodo en que se realizaron los muestreos. Esto permitirá encontrar una relación matemática que las relacione; para luego extrapolarla o interpolarla de acuerdo a la información de caudales líquidos diarios disponibles. El peso específico del sedimento en suspensión es considerado en esta investigación con un valor de 1.25 ton $/ \mathrm{m}^{3}$. 
Plano 1. Ubicación de los sitios de investigación

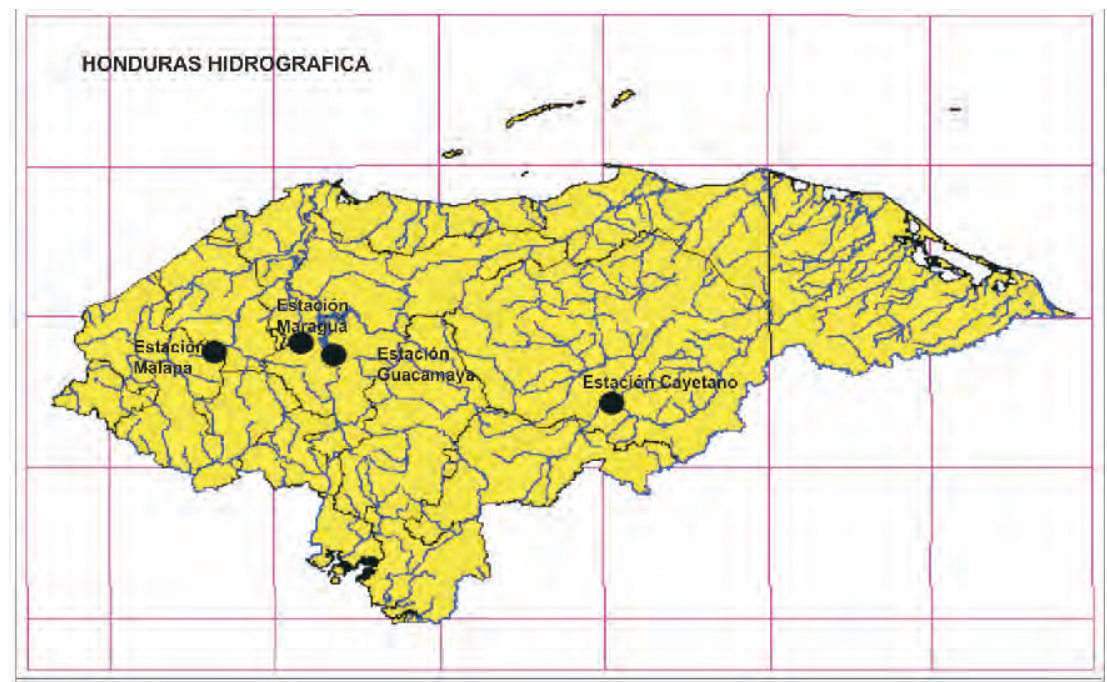

\section{APLICACIÓNYRESULTADOS}

\subsection{Generalidades}

La metodología fue aplicada a las estaciones de hidrométricas de Cayetano, Guacamaya, Malapa y Maragua. Se probó con cada una de ellas varios tipos de relación entre QL vs QS, a mencionar: relación lineal, relación polinomial de diferente orden, logarítmica, potencial y exponencial. Los mejores ajustes se obtuvieron utilizando un ajuste potencial, es decir $\mathrm{QS}=\mathrm{c}^{*} \mathrm{QL}{ }^{\wedge} \mathrm{n}$. Esta misma metodología fue aplicada a la información disponible de cada estación hidrométrica, permitiendo una uniformidad del tratamiento de la información y, por supuesto, una mejor interpretación de los resultados.

\subsection{Río Patuca}

Al sitio de la estación hidrométrica Patuca en Cayetano la cuenca correspondiente cubre un área hidrográfica de $10566.9 \mathrm{~km}^{2}$. La información recolectada de sedimento para esta investigación abarca el periodo de Marzo 2004 aAgosto 2007. La relación encontrada entre QL (m³/s) y QS (ton/día) es la siguiente:

\section{$Q S=0.0199{ }^{*} Q^{\wedge} 2.3902$}


El coeficiente de correlación determinado es de 0.94. Ver en Anexo Figura 1. En esta figura se muestra el ploteo de la información en Log-Log y el trazado de la ecuación que relaciona ambas variables. Utilizando los caudales líquidos diarios para el periodo 2004 y 2006 y la ecuación encontrada, el promedio obtenido de los rendimientos es de 133.445 (ton/año/km²), y lo concerniente a las pérdidas de suelo de $0.167 \mathrm{~mm} /$ año.

Como la estación hidrométrica de Cayetano tiene información de caudales líquidos desde 1973, la ecuación QS = f(QL) fue extrapolada de 1973 al 2006, haciendo un total de datos de 33 años. El Cuadro 1 muestra los resultados obtenidos.

En el Cuadro 1 (ver Anexo) se observa los rendimientos y pérdida de suelo para cada año con su respectivo promedio y total Dicho promedio corresponde a los sedimentos en suspensión y el total es la suma de los sedimentos en suspensión más el de fondo. De acuerdo al texto Diseño de Pequeñas Presas, publicado por el Bureau of Reclamation, no se dispone de información, el sedimento de fondo puede ser representado por el 10 al $15 \%$ del sedimento en suspensión. En nuestro caso consideramos un $15 \%$.

\subsection{Río Humuya}

Al sitio de la estación hidrométrica Humuya en Guacamaya, la cuenca correspondiente cubre una cuenca hidrográfica de $2570.0 \mathrm{~km}^{2}$. La información recolectada para esta investigación abarca el periodo de Agosto 1992 a Noviembre 2002. La relación encontrada entre QL ( $\mathrm{m}^{3} / \mathrm{s}$ ) y QS (ton/día) es la siguiente:

\section{$Q S=0.0704^{*} Q L^{\wedge} 2.5492$}

Con un coeficiente de correlación de 0.90 .

La Figura 2 muestra el ploteo de la información en Log-Log y el trazado de la ecuación que relaciona ambas variables. Para el periodo 1992 - 2002 el rendimiento obtenido fue de 700.368 ton $/$ año $/ \mathrm{km}^{2}$ y la pérdida de suelo de 0.875 $\mathrm{mm} / \mathrm{año.}$

Como se pudo disponer de caudales diarios de la estación hidrométrica de Guacamaya desde 1987 al 2006 la ecuación fue también aplicada para dicho periodo. Ver Figura 2, en anexo. 


\subsection{Río Maragua}

Al sitio de la estación hidrométrica Maragua en Maragua la cuenca correspondiente cubre una cuenca hidrográfica de $252.3 \mathrm{~km}^{2}$. La información recolectada para esta investigación abarca el periodo de Agosto 1992 a Noviembre 2004. La relación encontrada entre QL (m³/s) y QS (ton/día) es la siguiente:

\section{$Q S=0.0888^{*} Q L^{\wedge} 2.4966$}

Con un coeficiente de correlación de 0.933.

La Figura 3 muestra el ploteo de la información en Log-Log y el trazado de la ecuación que relaciona ambas variables. Para el periodo 1992 - 2004 el rendimiento obtenido fue de 122.527 ton $/ a n ̃ o / \mathrm{km}^{2}$ y la pérdida de suelo de 0.153 mm/año.

Como se pudo disponer de caudales diarios de la estación hidrométrica de Maragua desde 1988 al 2006, la ecuación fue también aplicada para dicho periodo. Figura 3 en anexo.

\subsection{Río Malapa}

Al sitio de la estación hidrométrica Malapa en Malapa la cuenca correspondiente cubre una cuenca hidrográfica de 110.33 km². La información recolectada para esta investigación cubre el periodo de 1991 al 2006. La relación encontrada entre QL $\left(\mathrm{m}^{3} / \mathrm{s}\right)$ y QS (ton/día) es la siguiente:

\section{$Q S=0.6833^{*} Q^{\wedge} 1.7487$}

Con un coeficiente de correlación de 0.90 .

La Figura 4 muestra el ploteo de la información en Log-Log y el trazado de la ecuación que relaciona ambas variables. Para el periodo 1991 - 2006 el rendimiento obtenido fue de 71.509 ton/año/ $/ \mathrm{km}^{2}$ y la pérdida de suelo de 0.089 mm/año.

Como se pudo disponer de caudales diarios de la estación hidrométrica de Maragua desde 1988 al 2006 la ecuación fue también aplicada para dicho periodo. Figura 4 en anexo. 


\section{ANÁLISIS E INTERPRETACIÓN}

Debido que no se tiene una serie larga de información de sedimento en suspensión se trató de utilizar toda la información que fue posible obtener; esto evitó seleccionar un periodo común de análisis para cada estación investigada. A parte de esto se pudo observar que por lo general la mayor cantidad de información es obtenida en los meses secos, provocando esto una subestimación de los rendimientos (ton/año/ $\mathrm{km}^{2}$ ) y pérdida de suelo (mm/año); por ejemplo, los casos más notorios son los resultados obtenidos en los ríos de Malapa (afluente al embalse de la hidroeléctrica El Níspero) y Maragua (afluente al embalse El Cajón. Según consultas realizadas en la ENEE, estos ríos tienen un mayor rendimiento y pérdida de suelo.

El extrapolar las relaciones hacia años anteriores no nos asegura que el comportamiento de los sedimentos sea iguales a los años recientes. Sin embargo sería interesante conocer la evolución de los rendimientos a través de periodos anteriores. Si comparamos las relaciones obtenidas $Q S=f(Q L)$ (ver Figura 5) se observa que las tendencias entre Guacamaya y Maragua son muy parecidas y, de igual forma, la de Cayetano se asemeja a Malapa. En el caso de Maragua y Guacamaya ambos son afluentes al embalse El Cajón. La coincidencia entre Cayetano y Malapa aparentemente es estadística De acuerdo a la información disponible, las estaciones de Guacamaya y Cayetano son las que presentaron mayor número de información, ya que cubren cuencas de mayor amplitud que las otras dos. Al estado actual de la información, y para esta investigación, los resultados obtenidos en Cayetano y Guacamaya pueden considerarse interesantes parajuzgar la hipótesis planteada.

En esta investigación la cuenca del río Humuya en Guacamaya queda como representativa para la zona central del país y la de Cayetano para la zona oriental. Los resultados obtenidos en los rendimientos muestran que ambas zonas tienen un rendimiento que cae entre 500 y 1000 ton/año/km². Figura 5 en anexo.

\section{CONCLUSIONES}

De los resultados obtenidos y de su interpretación se concluye lo siguiente:

a) Los datos disponibles de sedimento obtenido por muestreo integral, de las estaciones analizadas en la presente investigación han sido muestreadas en su mayor parte durante los meses secos. De igual forma la frecuencia de muestreo y su cantidad es variable en cada estación hidrométrica. 
b) La extrapolación hacia años anteriores de las relaciones $Q S=f(Q L)$ no son completamente válidas porque el sedimento no depende únicamente del caudal líquido sino también de otras variables, a mencionar como ejemplo suelo y cobertura vegetal.

c) Las cuencas de los ríos Malapa y Maragua necesitan una mayor cantidad de información para analizar sus rendimientos y pérdida de suelo.

d) De acuerdo al objetivo, a la cantidad y calidad de información disponible durante el desarrollar de la investigación y aceptando que las extrapolaciones favorecen en disponer de una mayor cantidad de información sin asegurar su trascendencia en el tiempo y que el número de muestra es pequeño, se concluye que la hipótesis planteada es aceptada; es decir, que los rendimiento de sedimento de la zona central y oriental del país se encuentran entre 500 y 1000 ton/año/km².

\section{AGRADECIMIENTO}

Se agradece a la Empresa Nacional de Energía Eléctrica del país por haber proporcionado la información de Caudales diarios y los Sedimentos diarios de las estaciones Malapa, Maragua, Guacamaya y Cayetano. Las que sirvieron de base para esta investigación; sin cuya información, hubiera sido imposible llevarla a cabo.

\section{REVISIÓN BIBLIOGRAFÍA}

Ávalos Lingán, Roberto. (2007). Análisis y Producción de Sedimento para el Proyecto Patuca III. Tegucigalpa: Publicación de la ENEE

Consultora GERACON (1997). Estudio de Factibilidad Proyecto Patuca III. Publicado en 1997. Tegucigalpa, Honduras Publicación de la ENEE.

Consultora LAHMEYER. (1991). Estudio de Factibilidad de los Proyectos Naranjito y Remolino-Sico. Tegucigalpa, Honduras. Publicación de la ENEE.

ENEE y Motor - Columbus. (1973) Proyecto El Cajón. Estudio de Factibilidad. Volumen 2. Publicado en Octubre 1973. Tegucigalpa, Honduras.

Secretaria de Recursos Naturales y Ambiente. (1991). Balance Hídrico de Honduras, Tegucigalpa, Honduras. Publicación de la Dirección General de Recursos Hídricos 


\section{ANEXO (Figuras)}

FIGURA 1: Estación Cayetano. Gráfico de QS vs QL

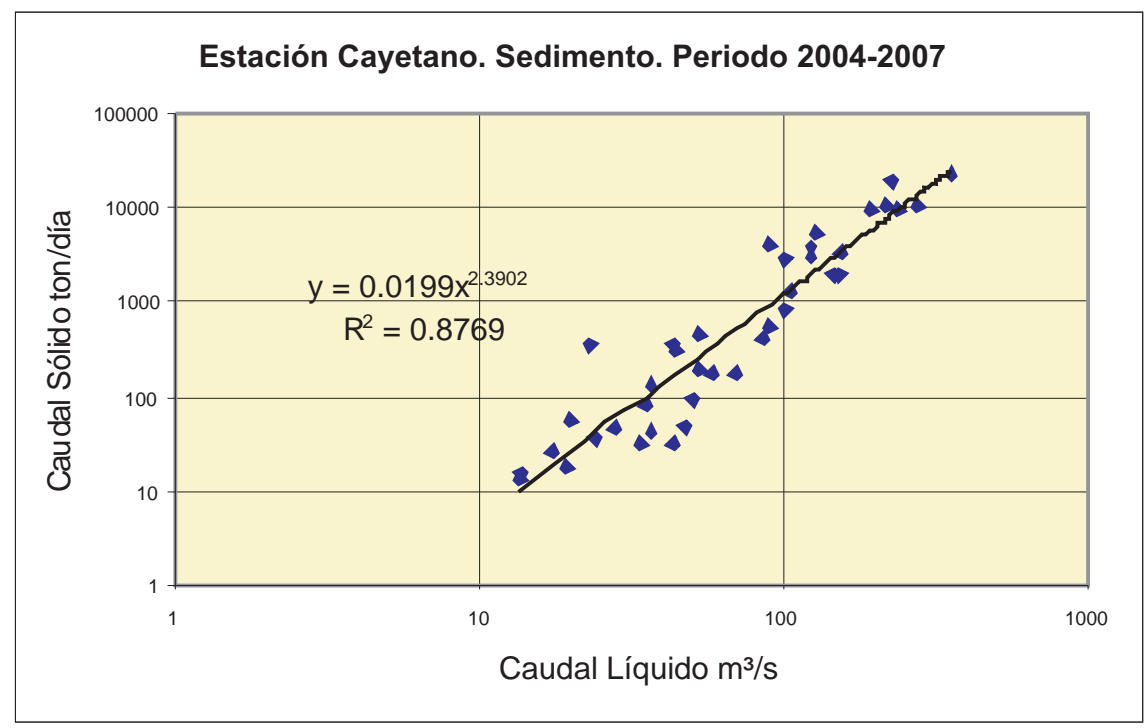

FIGURA 2: Estación Guacamaya. Ploteo Qs vs QL

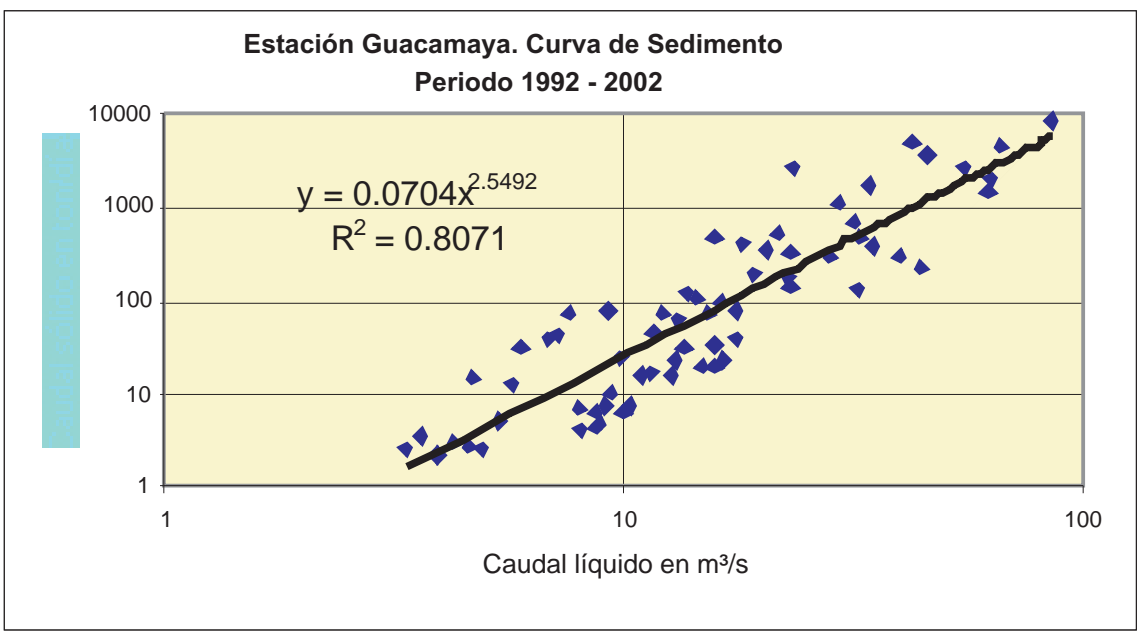


FIGURA 3: Estación Maragua. Ploteo de Qs vs QI

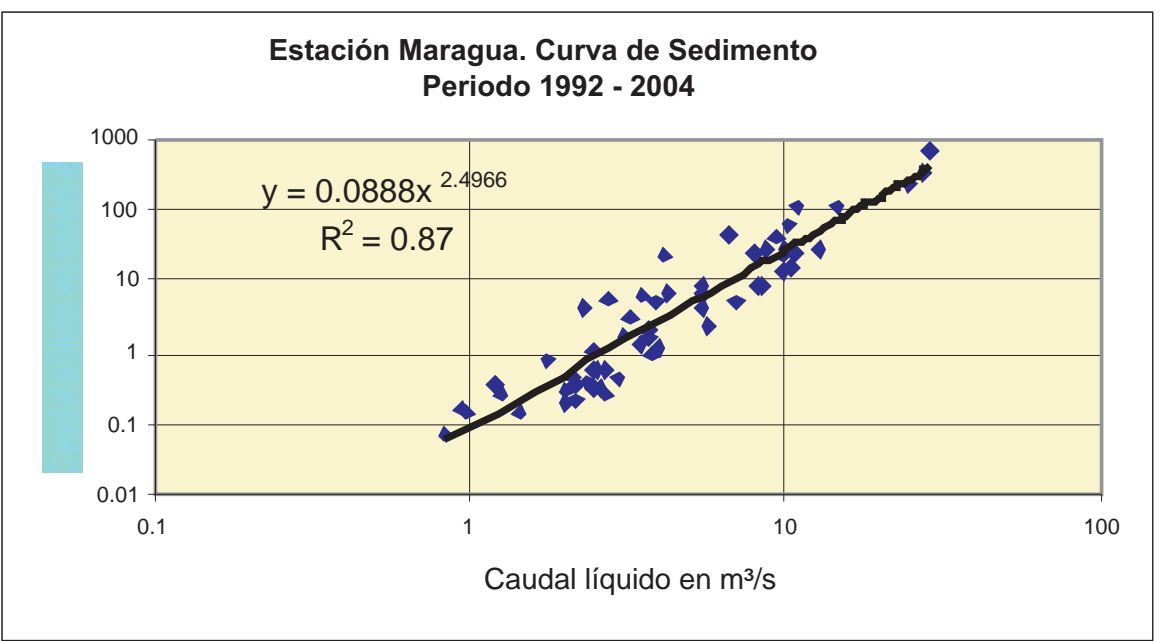

FIGURA 4: Estación Malapa. Ploteo de Qs vs QI

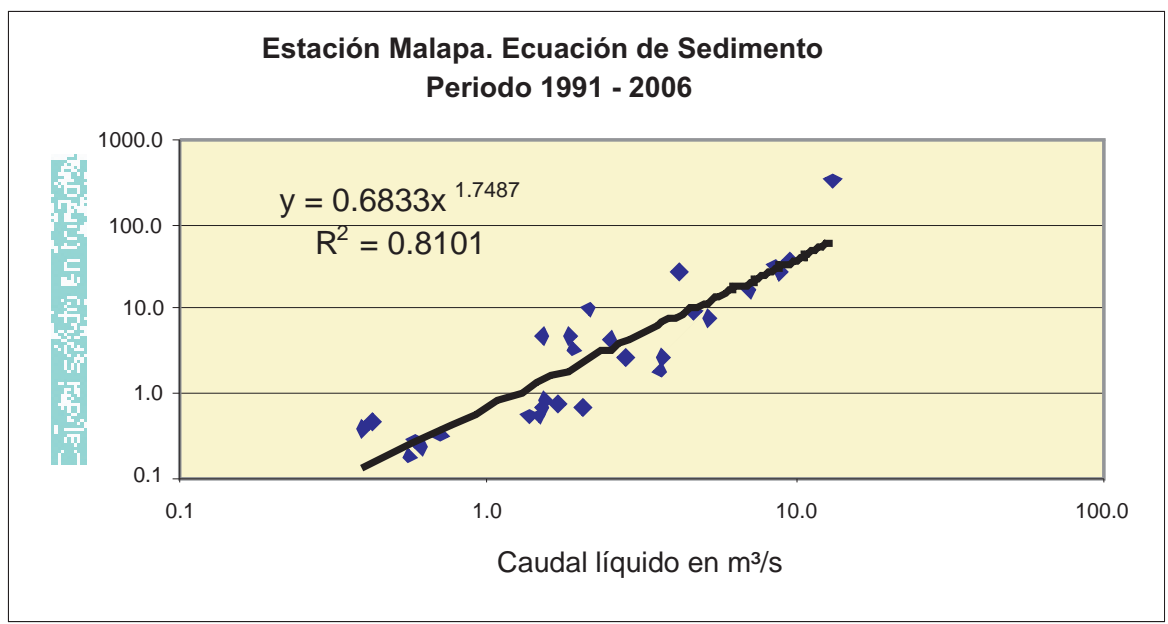


FIGURA 5: Comparación de relaciones $\mathrm{Qs}=\mathrm{f}(\mathrm{Q} \mathrm{l})$

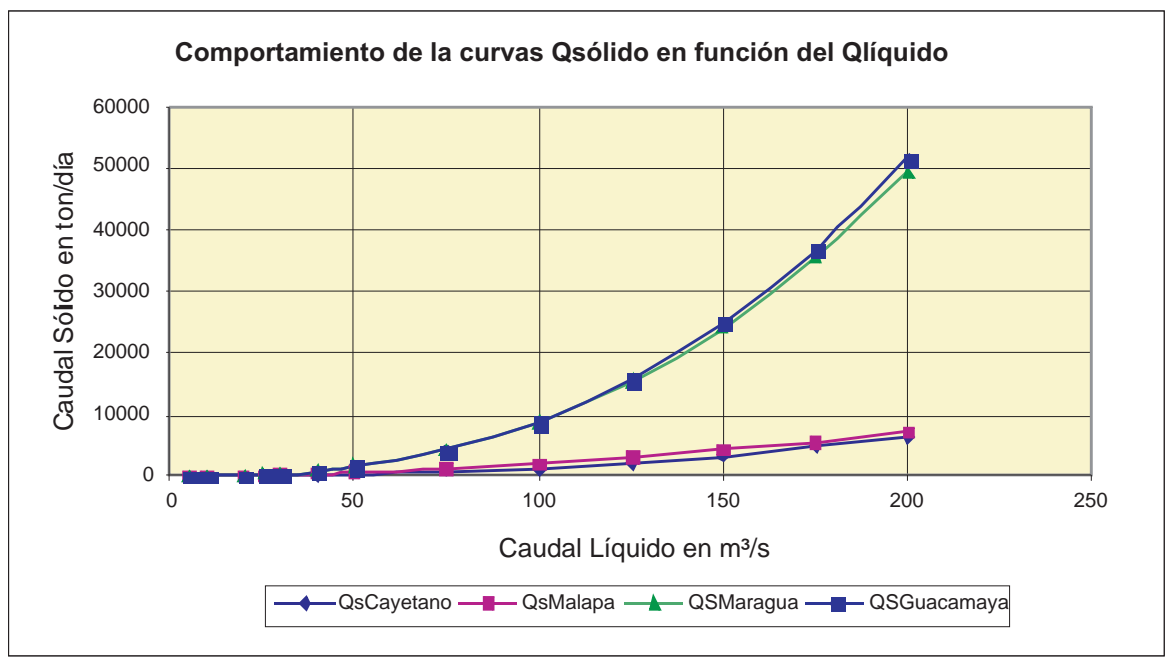




\section{ANEXO (Cuadros)}

CUADRO 1: Cuenca patuca en estación Cayetano. Rendimientos y Perdida de suelo

\begin{tabular}{|c|c|c|}
\hline \multirow{2}{*}{ Año } & Rendimiento & Pérdida Suelo \\
\hline & (ton/año/km²) & (mm/año) \\
\hline 1973 & 111.263 & 0.139 \\
\hline 1974 & 147.750 & 0.185 \\
\hline 1975 & 859.247 & 1.074 \\
\hline 1976 & 137.662 & 0.172 \\
\hline 1977 & 215.518 & 0.269 \\
\hline 1978 & 326.393 & 0.408 \\
\hline 1979 & 2284.035 & 2.855 \\
\hline 1980 & 554.592 & 0.693 \\
\hline 1981 & 372.186 & 0.465 \\
\hline 1982 & 623.205 & 0.779 \\
\hline 1983 & 301.777 & 0.377 \\
\hline 1984 & 304.238 & 0.380 \\
\hline 1985 & 70.835 & 0.089 \\
\hline 1986 & 527.349 & 0.659 \\
\hline 1987 & 407.178 & 0.209 \\
\hline 1988 & 635.954 & 0.795 \\
\hline 1989 & 260.183 & 0.325 \\
\hline 1990 & 314.737 & 0.393 \\
\hline 1991 & 721.555 & 0.902 \\
\hline 1992 & 291.764 & 0.365 \\
\hline 1993 & 1154.212 & 1.443 \\
\hline 1994 & 128.703 & 0.161 \\
\hline 1995 & 1409.723 & 1.762 \\
\hline 1996 & 396.404 & 0.496 \\
\hline 1997 & 394.449 & 0.493 \\
\hline 1998 & 5098.543 & 6.373 \\
\hline 1999 & 1463.812 & 1.830 \\
\hline 2000 & 74.677 & 0.093 \\
\hline 2001 & 24.651 & 0.031 \\
\hline 2001 & 141.861 & 0.177 \\
\hline 2003 & 114.705 & 0.143 \\
\hline 2004 & 69.254 & 0.087 \\
\hline 2005 & 282.806 & 0.354 \\
\hline 2006 & 48.285 & 0.060 \\
\hline $\begin{array}{c}\text { Promedio } \\
\text { S. Fondo }(15 \%)\end{array}$ & 596.162 & 0.745 \\
\hline Total & 685.586 & 0.857 \\
\hline
\end{tabular}


CUADRO 2: Rio Humuya en la estación Guacamaya. Rendimiento y Pérdida de suelo

\begin{tabular}{|c|c|c|}
\hline \multirow{2}{*}{ Año } & Rendimiento & Perdida Suelo \\
\cline { 2 - 3 } & (ton/año/km ${ }^{2}$ ) & (mm/año) \\
\hline 1987 & 150.6035798 & 0.188254475 \\
\hline 1988 & 1554.783772 & 1.943479714 \\
\hline 1989 & 630.8049274 & 0.788506159 \\
\hline 1990 & 709.4182196 & 0.886772774 \\
\hline 1991 & 308.1359937 & 0.385169992 \\
\hline 1992 & 2038.584667 & 2.548230833 \\
\hline 1994 & 305.5494008 & 0.381936751 \\
\hline 1995 & 240.4778871 & 0.300597359 \\
\hline 1996 & 1791.775612 & 2.239719515 \\
\hline 1997 & 557.7196896 & 0.697149612 \\
\hline 1998 & 373.4576691 & 0.466822086 \\
\hline 2000 & 1800.001537 & 2.250001921 \\
\hline 2001 & 340.7901051 & 0.425987631 \\
\hline 2002 & 74.95226168 & 0.093690327 \\
\hline 2003 & 132.1481716 & 0.165185215 \\
\hline 2004 & 217.5991135 & 0.271998892 \\
\hline 2005 & 141.0399826 & 0.176299978 \\
\hline 2006 & 1608.406153 & 2.010507692 \\
\hline Promedio & 957.2654461 & 1.196581808 \\
\hline S. Fondo (15\%) & 733.343 & 0.917 \\
\hline Total & 843.344 & \\
\hline
\end{tabular}

CUADRO 3: Rio Maragua en la estación Maragua. Rendimiento y Pérdida de suelo

\begin{tabular}{|c|c|c|c|}
\hline \multirow{2}{*}{ Año } & Sedimento & Rendimiento & Perdida Suelo \\
\hline & (ton/año) & (ton/año/km²) & (mm/año) \\
\hline 1988 & 26733.43151 & 105.9589041 & 0.13244863 \\
\hline 1989 & 8795.515522 & 34.86133778 & 0.043576672 \\
\hline 1990 & 42239.01421 & 167,4158312 & 0.209269789 \\
\hline 1991 & 2239.815169 & 8.877586878 & 0.011096984 \\
\hline 1992 & 32695.24362 & 129.5887579 & 0.161985947 \\
\hline 1993 & 27199.12729 & 107.8047059 & 0.134755882 \\
\hline 1994 & 41713.27926 & 165.3320621 & 0.206665078 \\
\hline 1995 & 50322.53639 & 199.4551581 & 0.249318948 \\
\hline 1996 & 42045.32421 & 166.648134 & 0.208310167 \\
\hline 2001 & 29721.49783 & 117.802211 & 0.147252764 \\
\hline 2002 & 3636.048358 & 14.41160665 & 0.018014508 \\
\hline 2003 & 6805.769304 & 26.97490806 & 0.033718635 \\
\hline 2004 & 7794.927326 & 30.89547097 & 0.038619339 \\
\hline 2005 & 55939.75653 & 221.7192094 & 0.277149012 \\
\hline 2006 & 41672.1907 & 165.1692061 & 0.206461508 \\
\hline $\begin{array}{l}\text { Promedio } \\
\text { S. Fondo }(15 \%)\end{array}$ & $\begin{array}{l}27970.23182 \\
4195.534772\end{array}$ & 110.861 & 0.139 \\
\hline$\frac{\text { Fondo }(15 \%)}{\text { Total }}$ & $\begin{array}{l}4195.534 / / 2 \\
32165.76659\end{array}$ & 127.490 & 0.159 \\
\hline
\end{tabular}

Nota: $\quad$ M Serie completada porque no tiene información en algunos meses del período seco. 
CUADRO 4: Rio Malapa en la estación Malapa. Rendimiento y Pérdida de suelo

\begin{tabular}{|c|c|c|c|c|}
\hline \multirow{2}{*}{ Año } & Sedimento & Meses & Pérdida Suelo & Rendimiento \\
\hline & (ton/año) & Faltantes & (mm/año) & (ton/año/km²) \\
\hline 1988 & 10514.009 & & 0.119 & 95.296 \\
\hline 1989 & 11199.178 & & 0.127 & 101.506 \\
\hline 1990 & 10222.571 & & 0.116 & 92.654 \\
\hline 1991 & 5494.083 & & 0.062 & 49.797 \\
\hline 1992 & 5588.366 & & 0.063 & 50.651 \\
\hline 1993 & 4491.765 & & 0.051 & 40.712 \\
\hline 1994 & 2872.759 & & 0.033 & 26.038 \\
\hline 1995 & 24006.000 & & 0.272 & 217.584 \\
\hline 1996 & 10724.673 & & 0.122 & 97.205 \\
\hline 1997 & 4575.768 & 10,11 y12 & & \\
\hline \multicolumn{5}{|l|}{1998} \\
\hline \multicolumn{5}{|l|}{1999} \\
\hline \multicolumn{5}{|l|}{2000} \\
\hline \multicolumn{5}{|l|}{2001} \\
\hline \multicolumn{5}{|l|}{2002} \\
\hline 2003 & 3666.668 & 1, 2 y 3 & & \\
\hline 2004 & 485.531 & del 7 al 12 & & \\
\hline 2005 & 4621.396 & $1,2,3,4,5$ y 8 & & \\
\hline 2006 & 8938.989 & & 0.101 & 81.020 \\
\hline Suma & 94052.392 & & & \\
\hline $\begin{array}{l}\text { Promedio } \\
\text { S. Fondo }(10 \%)\end{array}$ & $\begin{array}{c}9405.239 \\
940.524\end{array}$ & & 0.107 & 85.246 \\
\hline Total & 10345.763 & & 0.117 & 93.771 \\
\hline
\end{tabular}

